\title{
Leveraging latent persistency in United States patent and trademark applications to gain insight into the evolution of an innovation-driven economy
}

Iraj Daizadeh1

How to cite: Daizadeh, I. (2021) Leveraging latent persistency in United States patent and trademark applications to gain insight into the evolution of an innovation-driven economy. Iberoamerican Journal of Science Measurement and

Communication; $1(3)$,

1-23. https://doi.org/10.47909/ ijsmc.32

Received: 8-12-2020

Accepted: 27-06-2021

Handling Editor: Ulf Sandströme

Copyright: () 2021 Daizadeh. This is an open access article distributed under the terms of the CC BY-NC 4.0 license which permits copying and redistributing the material in any medium or format, adapting, transforming, and building upon the material as long as the license terms are followed.

\section{ABSTRACT}

Objective. An understanding of when one or more external factors may influence the evolution of innovation tracking indices (such as US patent and trademark applications (PTA)) is an important aspect of examining economic progress/ regress. Using exploratory statistics, the analysis uses a novel tool to leverage the long-range dependency (LRD) intrinsic to PTA to resolve when such factor(s) may have caused significant disruptions in the evolution of the indices, and thus give insight into substantive economic growth dynamics.

Design/Methodology/Approach. This paper explores the use of the Chronological Hurst Exponent (CHE) to explore the LRD using overlapping time windows to quantify long-memory dynamics in the monthly PTA time series spanning 1977 to 2016.

Results/Discussion. The CHE is found to increase in a clear S-curve pattern, achieving persistence $(\mathrm{H} \sim 1)$ from non-persistence $(\mathrm{H} \sim 0.5)$. For patents, the inflection occurred over a span of 10 years (1980-1990), while it was much sharper (3 years) for trademarks (1977-1980).

Conclusions/Originality/Value. This analysis suggests (in part) that the rapid augmentation in R\&D expenditure and the introduction of the various patent-directed policy acts (e.g., Bayh-Dole, Stevenson-Wydler) are the key factors behind persistency, latent in PTA. The post-1990's exogenic factors seem to be simply maintaining the high degree and consistency of the persistency metric. These findings suggest investigators should consider latent persistency when using these data and the $\mathrm{CHE}$ may be an important tool to investigate the impact of substantive exogenous variables on growth dynamics.

Keywords: Innovation, Hurst, trademarks, patents, persistency, economy

1 Takeda Pharmaceuticals, United States. Email: iraj.daizadeh@takeda.com, ORCID: 0000-0003-3648-023X. 


\section{INTRODUCTION}

Intellectual property (IP)-based metrics (initially patents and more recently trademarks) have been used as proxy measures of global politico-socio-economic innovative behavior [on the micro (firm)-, meso (research institution, cluster)-, and/or macro (country/regional)-level] for decades (see, e.g., Dziallas and Blind, 2019). These and related indices focus on the time-dependent ebbs and flows of absolute counts of observations and/or derivates thereof. Importantly, it is understood that these data integrate one or more extrinsic forces and/or factors that are either directly or indirectly influenced the chronological evolution of these data in some way (see, e.g., Daizadeh, 2007, 2009, 2021). Indeed, a vast majority of this research either test or generate a hypothesis of how such extrinsic impulses - such as the promulgation of a specific policy framework, gross effects of research and development expenditures, sector-specific technology dynamics (e.g., disruptive versus incremental) - may affect the time course of these IP-metrics (see, e.g., Grimaldi and Cricelli, 2020; Flikkema, et al., 2015; Daizadeh, 2007, 2021). Unfortunately, to the author's knowledge, there are limited-to-no inquiries exploring the intrinsic make-up of these time-series data.

Even in the simplest of cases, the interpretative potential of time series data, such as those of patents and trademarks, may be challenging, however, due to their intrinsic behavior (s) (see, e.g., Cheng et al., 2015). Importantly and simultaneously, some of these properties are also of interest due to the potential to elucidate interesting dynamics. For example, memory characteristics across length scales (short, medium, and long) suggest the existence of various economic cycles (see, e.g., Alvarez-Ramirez et al., 2020). Concordantly, these same memory characteristics can obfuscate true signal detection, due to - for example - mistakenly considering long-memory and non-stationary complex dynamics (see, e.g., Saha et al., 2020). Other properties such as linearity, normality, and seasonality of the time series may further exacerbate misinterpretations. Statistical time series practitioners exert great effort in developing methods that would increase the certitude of the interpretation by accommodating one or more of these properties. Resolving the suite of issues is the first step in establishing robust statistical approaches and increased confidence in model interpretability.

The Hurst exponent is a statistical time-series tool that has been used to better understand memory effects in time series data and has been applied to various fields including earth sciences (see, e.g., Slino, et al., 2020), economics (see, e.g., Wu and Chen, 2020), and others. While there are other ways, the Hurst exponent (a measurement of memory) is classically defined as $\mathrm{H} \sim \ln (\mathrm{R} / \mathrm{S}) \mathrm{t} / \ln (\mathrm{t})$, where $\mathrm{R}$ and $\mathrm{S}$ are the rescaled range and standard deviation, respectively, and $\mathrm{t}$ is a time window. $\mathrm{An} \mathrm{H}=0.5$, an $\mathrm{H}<0.5$, and an $\mathrm{H}>0.5$ indicate a random walk (non-persistent), an anti-persistent, and a persistent (trend reinforcing) time series behavior, respectively (see, e.g., Mandelbrot and Wallis, 1968, 1969).

To the author's knowledge, there have not been any investigations of the Hurst exponent with regards to either patents or trademarks. In terms of patents, however, some elements of memory effects have been explored in the context of economic cycles by several authors (see, e.g., Korotayev, 2011; Haustein and Neuwirth, 1982, Alvarez-Ramirez, 2020, Epicoco, 2020, Daizadeh, 2021a). Korotayev et al (2011) demonstrated a Kondratieff (K)-wave pattern when investigating the dynamics of the annual number of global patents per million population from 1900 to 2008. As summarized by (Alvarez-Ramirez, 2020), Korotayev showed that patents presented with "a steady increase during the upswing phase of Kondratieff's cycle, and a pronounced decrease during the downswing phase." Haustein and Neuwirth (1982) found that "industrial production on patents with a lag of 9 years." More recently, Epicoco (2020) fitted the information and communications technologies cycle with that of the economy using patent and productivity data and proposed "the current productivity slowdown may be a 
signal that the economic system needs to change its leading technologies."

Carbone, Castelli, and Stanley (2004) proposed a time-dependent Hurst exponent based on a detrending moving average (DMA). Here, the Hurst exponent is a log-log slope of the DMA standard deviation against window (see equations 1 and 2 therein). The authors conclude from an analysis of artificial and observed time series of financial data that the time-variability is much "richer" than anticipated from a mono-fractal approach. To accommodate non-stationary effects, the work was subsequently extended with the aid of detrended fluctuation analysis over non-overlapping window lengths by Alverez-Ramirez and colleagues (2020). Effectively, these approaches to time-dependent Hurst exponent calculations are model parameterized.

In this work, and as described in the Methodology section below, a non-model parameterized chronological Hurst exponent $(\mathrm{CHE})$ is proposed that when applied to a given time series may identify significant changes in the persistency of memory. The method is straightforward to implement since it simply uses a standard estimate of the Hurst exponent calculated from the initial time point to month a, where a is a monthly increment. The output of the CHE calculation is described as the time series plot of each of the Hurst exponents, allowing a qualitative view of the Hurst exponent over a given time period (see Methods). This approach allows for an arbitrary method to calculate the Hurst exponent while taking into regard the nuances of the time series. Here, the method is applied to US patent and trademark applications (PTA) from 1977-2016. The date range selected is chronologically broad (a period of over 40 years) and used in prior work, and thus presented here. Interested readers may extend the data range accordingly, as all data and $\mathrm{R}$ Programs used for this manuscript are available (Appendix; Daizadeh, 2021b).

As described in the Results section below, from the application of this novel tool to PTA, it is found that the $\mathrm{CHE}$ evolves in a highly descriptive and idiosyncratic S-like pattern: from non-persistent $(\mathrm{H} \sim 0.5)$ to saturation (trend reinforcing persistent $(\mathrm{H} \sim 1)$ level via a quickly evolving inflection period (see Figures 1-3). For patents, the inflection occurred over a span of 10 years (1980-1990), while it was much sharper (3 years) for trademarks (1977-1980). As will be further discussed below, these findings suggest: investigators should consider latent persistency when using these data; exogenous factors after the identified inflection points for these indices have only incrementally strengthened intrinsic memory; and, the CHE may be an important tool to investigate the impact of substantive exogenous variables on growth dynamics. Qualitative correlation of the timing of the inflection points for patents and trademarks suggests the importance of research and development expenditure.

\section{METHODOLOGY}

The data were comprised of the monthly number of US patent applications (Patents) and the monthly number of US trademarks filings (Trademarks) (together, PTA) from 1977 to 2016, and were obtained from the United States Patent and Trademark Office (USPTO) as described below:

- Patents:

- Website: http://patft.uspto.gov/netahtml/PTO/search-adv.htm

- Search pattern: Application Filing Date: "APD/MM/\$/YYYY"

- Trademarks:

- Website: http://tmsearch.uspto.gov/bin/gate.exe?f=tess\&state=4804:57thz4.1.1

- Search pattern: Filing Date: "(YYYYMM\$)[FD]" 
Note: MM/YYYY is the $2 / 4$ digital representation for month/year. The two searches resulted in 472 datapoints - representing monthly observations over the period of study (approximately 40 years) - for each variable and imported into $\mathrm{R}$ for processing (Appendix; Daizadeh, 2021b).

The methodology followed standard implementation, and default parameters were used throughout. The general algorithm for the analysis is as follows:

- Load time series (R package 'tseries' (Trapletti and Hornik, 2019)), identify and replace outliers with an average of prior and posterior-month values ( $R$ package 'tsoutliers' (López-de-Lacalle, 2019). Note: 3 outliers were determined for Trademarks (September 1982; November 1989; and June 1999) and 4 for Patents (September 1982, June 1995, October 2007, and March 2013).

- Calculate descriptive statistics [including standard deviation, kurtosis, and skew (R package 'moments' (Komsta and Novomestky, 2015)] and auto/serial correlation (base R package).

- Calculate intrinsic variables: normality (R package 'nortest' (Gross and Ligges, 2015)), stationarity (R package 'forecast' (Hyndman, et al., 2020; Hyndman and Khandakar, 2008; R package 'aTSA' (Qiu, 2015)), seasonality (R package 'seastests' (Ollech, 2019)), and non-linearity (R package 'nonlinearTseries' (Garcia, 2020))

- Calculate chronological Hurst exponent: Determine Hurst exponent based on Hyndman implementation (R package 'tsfeatures' (Hyndman et al, 2020)) using the following algorithm:

- - for (i in start:end) \{ hurst-IP[i] <- hurst (time[1:(1+i*1)]) \}, where IP is either Trademarks or Patents; start $=$ September, 1977; end $=$ December 2016; $I=$ monthly increments

- Note: The Hyndman approach - one of several methods to calculate (estimate) the Hurst Exponent (Shang, 2020) - is defined as 0.5 plus the maximum likelihood estimation of the fractional differencing order (see Hyndman et al, 2020); thus, it has properties that differ than Hurst's original definition (e.g., no singularities at certain scales). In principle, any approach should produce qualitatively the same result as that outline above, albeit additional work is required to confirm the approaches sensitively.

\section{RESULTS AND DISCUSSION}

\section{General Statistics}

Generally, the time series were similar in structure with a general cobra-like structure (see upper graphs of Figures 1,2, and 3) and similarities in the shape of the distributions (e.g., approximately symmetric (skew) and platykurtic) (see Table 1). The time series showed clear long-memory tendency as presented in the auto and serial correlation functions with lag much great than 2. Lastly, both time series were non-normal, non-stationary [with a single difference $((t-1)-t)$ bringing them into stationarity - that is, integration of order 1 typical of econometric data], seasonal, and non-linear (see Table 2). 


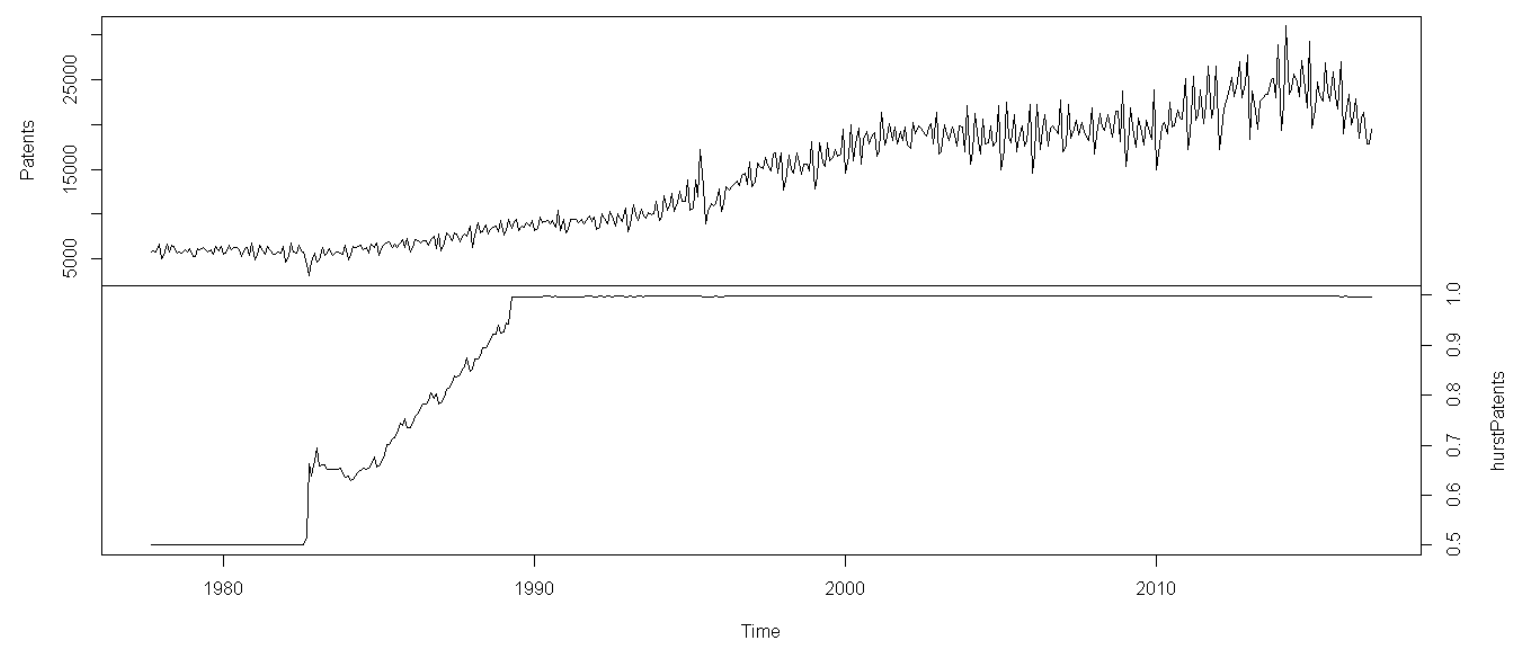

Figure 1. The monthly number of Patents (top graph) from 1977-2016 with its corresponding chronological Hurst values (bottom graph).

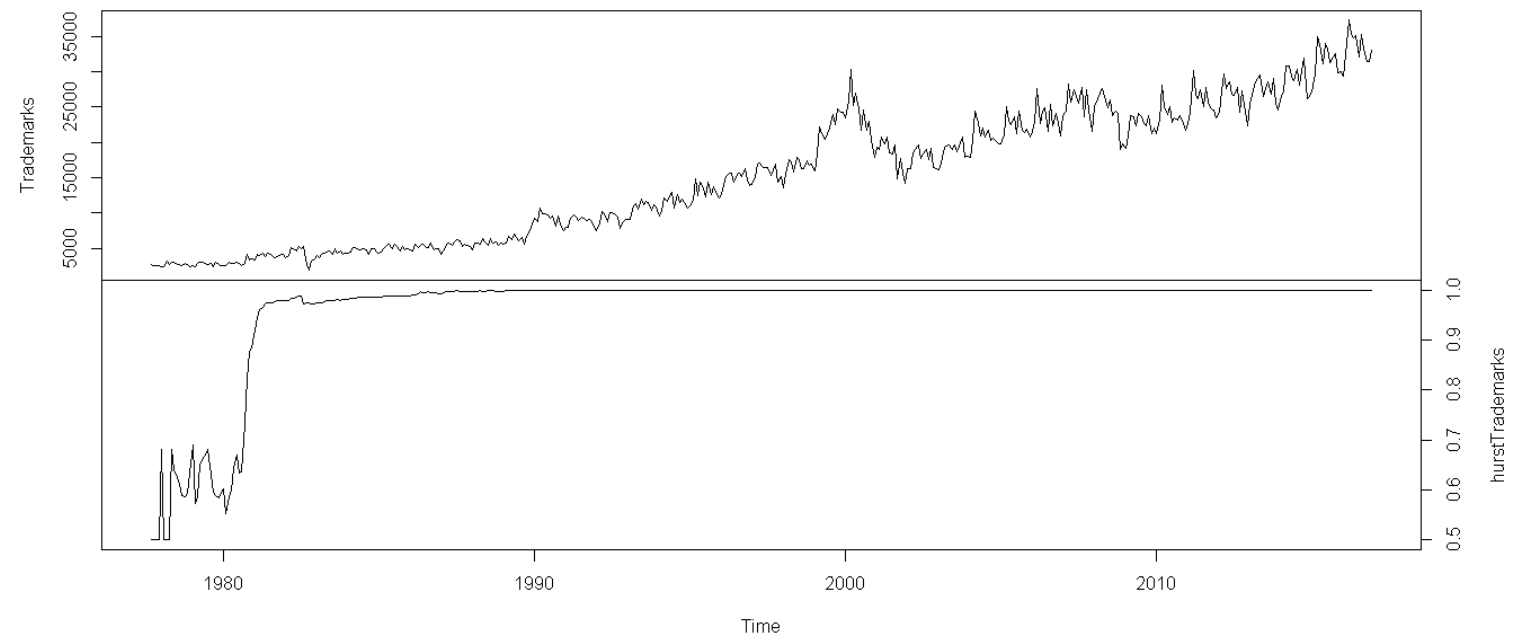

Figure 2. The monthly number of Trademarks (top graph) from 1977-2016 with its corresponding chronological Hurst values (bottom graph). 


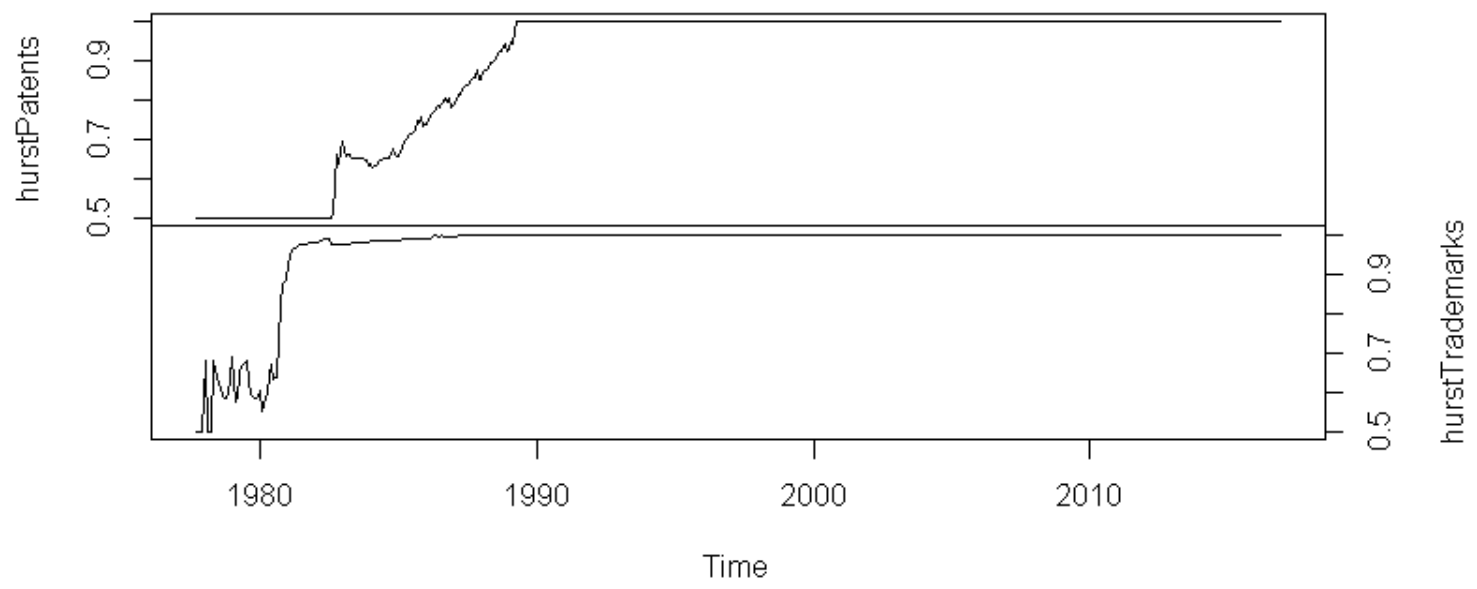

Figure 3. Comparison of chronological Hurst values between Patents and Trademarks.

Table 1. Descriptive statistics of Patents and Trademarks.

\begin{tabular}{lll}
\hline & Patents & Trademarks \\
\hline Minimum & 3134 & 1895 \\
1st Quantile & 7598 & 5537 \\
Median & 14468 & 15456 \\
Mean & 13930 & 15276 \\
3rd Quantile & 19422 & 23574 \\
Maximum & 30969 & 37317 \\
Stand Deviation & 6523.347 & 9418.054 \\
Skewness & 0.1854975 & 0.2020644 \\
Kurtosis & 1.757654 & 1.763723
\end{tabular}

Table 2. General results of intrinsic parameters of Patents and Trademarks (see Appendix; Daizadeh, 2021b).

\begin{tabular}{lll}
\hline & Patents & Trademarks \\
\hline Normality & Non-normal & Non-normal
\end{tabular}




\begin{tabular}{lll}
\hline \multicolumn{1}{c}{ Patents } & Trademarks \\
\hline Stationarity & $\begin{array}{l}\text { Non-stationary (order of integration: } \\
1\end{array}$ & $\begin{array}{l}\text { Non-stationary (order of integration: } \\
1\end{array}$ \\
Seasonality & Seasonal & Seasonal \\
Non-linearity & Non-linear & Non-linear \\
\hline
\end{tabular}

\section{Chronological Hurst Exponent}

Examining Figures 1 and 2, the time evolution of PTA shows a general trend-reinforcing pattern, edging generally up with time. There are variations of course partly due to the identified seasonal effects. The Chronological Hurst Exponent (CHE) correspondingly evolves, with non-persistence $(\mathrm{H} 0.5)$ near the beginning of the time series (notice: this is where the absolute counts of the PTA are effectively static). As the time series evolves, the $\mathrm{CHE}$ quickly jumps to saturation $(\mathrm{H} \sim 1)$. A Hurst exponent at such a high relative value suggests that that the trend will continue to persist in the original time series - that is, the exponent reflects the similarity of prior absolute values of PTA to future values; in other words: as prior timepoints values edge up, it is likely that the next value will also be up. This is consistent with the long auto and serial correlations in the original time series.

Co-positioning PTA CHE (as in Figure 3), a similarity in S-structure is presented. The scaling regimes of are trichotomized in both time series into the following periods: (1) non-persistence (albeit for Trademarks mild persistence is observed with $\mathrm{H}$ values oscillating between 0.5 and 0.7 ); (2) time-varying persistence (an inflection, where $\mathrm{H}$ slopes from $0.5 / 0.6$ to near 1 ); and, (3) persistence $(\mathrm{H} \sim 1)$. Specifically, it is found that:

- Period 1: Weak to no persistence prior to 1980s:

- Patents: The flat $\mathrm{H}$ exponent, as shown in Figure 1 (lower graph) and Figure 3 (upper graph), reflects the neutral acceleration in the time series.

- Trademarks: The variation in $\mathrm{H}$ exponent $(\sim 0.5$ to $\sim 0.7)$ reflects a positive trajectory as depicted in Figures 2 (upper graph) and 3 in the reference time series.

- Period 2: A time-varying Hurst exponent up to and during the 1980s and 1990s

- Patents: The value of the $\mathrm{H}$ exponent began to grow with a rapid linear rate of approximately $0.1-0.2$ Hurst values per year, commencing with an explosive charge in the mid-1980s, and terminating with saturation before 1990 .

- Trademarks: The value of the $\mathrm{H}$ exponent grew at an exceptional rate at roughly 0.3 over two years, from effective baseline to near saturation by 1983.

- Period 3: Saturated persistence for trademarks beginning by 1983 and for patents in the 1990s throughout the duration of the reporting period 2016.

Focusing on the time-varying Period 2, while more work needs to be done to better our understanding of the dynamics in the 1980s and 1990s that affected these proxy measures of innovativeness, some thoughts present themselves for potentially testable causality hypotheses:

- Economic: Total spending on research and development grew from \$60B USD in 1975 to $\$ 100 \mathrm{~B}$ by 1985 , concomitantly while the contribution from industry to the percent of Gross Domestic Product rose from $1 \%$ to nearly $1.5 \%$ during that same time (see Figure 1 in Hunt, 1999). Indeed, patent activity (number of Patent Applications) also doubled grew from 100k 
(1980) to 200k (2000) (see Figure 2 in ibid). Could industrial R\&D expenditures directly drive the persistence of Patents and Trademarks?

- Policy: There were significant competitive policy initiatives during the 1980s including the Bayh-Dole Act (Patent and Trademark Law Amendments Act (Pub. L. 96-517, December 12, 1980), Public Law 96-480, Stevenson-Wydler Technology Innovation Act (as amended in 1986 and 1990), and others (see Table 1 in Coriat and Orsi, 2002). Was the persistency initiated by one or more of these policies (including, for example, new patentability types: software and/or business plans)?

- Technology dynamics: S-shaped growth for individual technological innovations may contribute to the overall Patent evolution dynamic (see, e.g., Anderson, 1999). A linear combination of such patent growths may be affecting the persistence of the overall Patents structure. To the author's knowledge, there is limited to no information on any S-shaped distribution for Trademarks, the potential for future queries. Do technology dynamics associated with innovation cumulatively affect persistence in the innovation metrics?

\section{CONCLUSION}

The goal for the use of a novel method - termed the chronological Hurst exponent (CHE) - to investigate long-term memory dynamics in time series data is to describe the overall persistency tend and, importantly, to identify the key dates in which there is a change in persistency (anti-persistence, non-persistence, persistence). Here, the CHE was applied to two innovative-tracking, intellectual property-driven data: the monthly numbers of patent and trademark applications. CHE is a simple to use method, trivially constructed in any programming language (Appendix; Daizadeh, 2021b) and easily applied to longitudinal data. Additional work is required to better understand the approach, including using different memory quantification tools (e.g., different versions of the Hurst exponent calculation) and different (longer) datasets with different characteristics.

In summary, this paper presents the first use of the CHE as well as the initial results of its application to PTA. The importance of persistency as an intrinsic factor of time series data should not be underestimated as effects such as trend-reinforcing behavior may lead to biased results when interpreting time-series data, as the momentum of the time series may cover salient investigative concerns (recall that the Hurst exponent is a measure of self-similarity). It is a hope of this work that investigators continue to examine the innovative accomplishments of the late $20^{\text {th }}$ century as well as consider persistency measures in current assessments of techno-economic progress.

\section{Conflict of Interest Statement}

The author is an employee of Takeda Pharmaceuticals; however, this work was completed independently of his employment. The views expressed in this article may not represent those of Takeda Pharmaceuticals.

\section{Statement of Data Consent}

The data generated during the development of this study has been included in the manuscript.

\section{References}

Alvarez-Ramirez, J.; Rodriguez, E.; Ibarra-Valdez, C. (2020) Medium-term cycles in the dynamics of the Dow Jones Index for the period 1985-2019. Physica A: Statistical Mechanics 
and its Applications. 546: 124017 https://doi.org/10.1016/j.physa.2019.124017

Anderson, B. (1999) The hunt for S-shaped growth paths in technological innovation: a patent study. J Evol Econ (1999) 9: 487-526 https://doi.org/10.1007/s001910050093

Carbone, A.; Castelli, G.; Stanley, H.E. (2004) Time-dependent Hurst exponent in financial time series. Physica A: Statistical Mechanics and its Applications. 344(1-2): 267-271 https://doi.org/10.1016/j.physa.2004.06.130

Cheng, C., Sa-Ngasoongsong, A., Beyca, O., Le, T., Yang, H., Kong, Z., \& Bukkapatnam, S. T. (2015). Time series forecasting for nonlinear and non-stationary processes: A review and comparative study. lie Transactions, 47(10), 1053-1071. https://doi.org/10.1080/ 0740817X.2014.999180

Coriat, B.; Orsi, F. (2002) Establishing a new intellectual property rights regime in the United States: Origins, content and problems. Research Policy 31:1491-1507. https://doi.org/ 10.1016/S0048-7333(02)00078-1

Daizadeh, I. (2007) Issued US patents, patent-related global academic and media publications, and the US market indices are inter-correlated, with varying growth patterns. Scientometrics 73(1): 29-36. https://doi.org/10.1007/s11192-007-1749-1

Daizadeh, I. (2009). An intellectual property-based corporate strategy: An R\&D spend, patent, trademark, media communication, and market price innovation agenda. Scientometrics 80 (3): 731-746. https://doi.org/10.1007/s11192-008-2105-9

Daizadeh, I. (2021). Trademark and patent applications are structurally near-identical and cointegrated: Implications for studies in innovation. Iberoamerican Journal of Science Measurement and Communication, 1(2). https://doi.org/10.47909/ijsmc.33

Daizadeh, I. (2021a). US FDA Drug Approvals are Persistent and Polycyclic: Insights into Economic Cycles, Innovation Dynamics, and National Policy. Therapeutic Innovation and Regulatory Science. https://doi.org/10.1007/s43441-021-00279-8

Daizadeh, I. (2021b). Chronological Hurst exponent elucidates latent persistency within patents and trademarks applications reflecting strength of innovation initiatives between 1977 and 2016. arXiv preprint arXiv:2101.02588.

Dziallas, M.; Blind, K. (2019). Innovation indicators throughout the innovation process: An extensive literature analysis. Technovation 80-81: 3-29. https://doi.org/10.1016/ j.technovation.2018.05.005

Epicoco, M. (2020). Technological Revolutions and Economic Development: Endogenous and Exogenous Fluctuations. J. Knowl. Econ. https://doi.org/10.1007/s13132-020-00671-z

Garcia, C.A. (2020) nonlinearTseries: Nonlinear Time Series Analysis. R package version 0.2.10. https://CRAN.R-project.org/package=nonlinearTseries

Grimaldi, M.; Cricelli, L. (2020). Indexes of patent value: a systematic literature review and classification, Knowledge Management Research \& Practice, 18:2, 214-233, https://doi.org/ 10.1080/14778238.2019.1638737 
Haustein H.D.; Neuwirth E. (1982) Long waves in world industrial production, energy consumption, innovations, inventions, and patents and their identification by spectral analysis. Technol. Forecast. Soc. Change, 22:53-89 https://doi.org/10.1016/0040-1625(82)90028-2

Hunt, R.M. (1999) Patent reform: a mixed blessing for the US economy. Business Review. Federal Bank of Philadelphia, November-December. Accessed via https://www.philadelphiafed.org/-/media/research-and-data/publications/business-review/ 1999/november-december/brnd99rh.pdf

Hyndman, R.; Kang, Y.; Montero-Manso, P.; Talagala, T.; Wang, E.; Yang, Y.; O'Hara-Wild, M. (2020) tsfeatures: Time Series Feature Extraction. $R$ package version 1.0.2. https://CRAN.R-project.org/package=tsfeatures

Komsta, L.; Novomestky, F. (2015). moments: Moments, cumulants, skewness, kurtosis and related tests. $\mathrm{R}$ package version 0.14 . https://CRAN.R-project.org/package=moments

Gross, J.; Ligges, U. (2015). nortest: Tests for Normality. R package version 1.0-4. https://CRAN.R-project.org/package=nortest

Hyndman, R.; Athanasopoulos, G.; Bergmeir, C.; Caceres, G.; Chhay, L.; O'Hara-Wild, M.; Petropoulos, F.; Razbash, S.; Wang, E.; Yasmeen, F. (2020). forecast: Forecasting functions for time series and linear models. R package version 8.12, http://pkg.robjhyndman.com/ forecast

Hyndman, R.J.; Khandakar, Y. (2008). "Automatic time series forecasting: the forecast package for R." Journal of Statistical Software, ${ }^{*} 26^{*}(3), 1-22$. http://www.jstatsoft.org/article/ view/v027i03

Korotayev, A.; Zinkina, J.; Bogevolnov, J.; (2011) Kondratieff waves in global invention activity (1900-2008). Technol. Forecast. Soc. Change, 78:1280-1284 https://doi.org/10.1016/ j.techfore.2011.02.011

López-de-Lacalle, J. (2019). tsoutliers: Detection of Outliers in Time Series. R package version 0.6-8. https://CRAN.R-project.org/package=tsoutliers

Mandelbrot, B.B.; Wallis, J.R. (1969) Noah, Joseph, and Operational Hydrology. Water Resources Research 4(5) https://doi.org/10.1029/WR004i005p00909

Mandelbrot, B.B.; Wallis, J.R. (1969) Robustness of the rescaled range R/S in the measurement of noncyclic long run statistical dependence. Water Resources Research 5(5) https://doi.org/10.1029/WR005i005p00967

Ollech, D. (2019). seastests: Seasonality Tests. $R$ package version 0.14.2. https://CRAN.R-project.org/package=seastests

Qiu, D. (2015). aTSA: Alternative Time Series Analysis. R package version 3.1.2. https://CRAN.R-project.org/package=aTSA

R Core Team (2019). R: A language and environment for statistical computing. R Foundation for Statistical Computing, Vienna, Austria. URL https://www.R-project.org/ Version 3.6.1 (2019-07-05) 
Saha, K.; Madhavan, V.; Chandrashekhar, G.R. (2020) Pitfalls in long memory research. Cogent Economics and Finance 8: $1733280 \mathrm{https} / / /$ doi.org/10.1080/23322039.2020.1733280

Shang, H. (2020). A Comparison of Hurst Exponent Estimators in Long-range Dependent Curve Time Series. Journal of Time Series Econometrics, 12(1). https://doi.org/10.1515/ jtse-2019-0009

Slino, M.; Scudero, S.; D’Alessandro, A. (2020). Stochastic models for radon daily time series: seasonality, stationarity, and long-range memory detection. Frontiers in Earth Sciences. https://doi.org/10.3389/feart.2020.575001

Trapletti, A.; Hornik, K. (2019). tseries: Time Series Analysis and Computational Finance. R package version 0.10-47.

Wu, K.; Chen, S.(2020) Long memory and efficiency of Bitcoin under heavy tails, Applied Economics, 52:48, 5298-5309. https://doi.org/10.1080/00036846.2020.1761942

\section{APPENDIX: R CODE FOR TRANSPARENCY AND REPRODUCIBILITY}

\# Start: R code with dataset \#

\#Trademarks

\#Go to TESS: http://tmsearch.uspto.gov/bin/gate.exe?f=tess\&state=4804:57thz4.1.1

\# Manually search and collect Number of Trademarks as follows:

\#By Filing Date: "(198712\$)[FD]" - Where 198712\$ is the \%Y\%m\$.

\#Patents

\#Go to PATFT: http://patft.uspto.gov/netahtml/PTO/search-adv.htm

\#By Application Filing Date: "APD/12/\$2018"

\#The patent and trademark filings data were collected from Sept 1977 to Dec 2018.

\#Confirm version of $\mathrm{R}$ :

$>$ citation()

R Core Team (2019). R: A language and environment for statistical computing. R Foundation for Statistical Computing, Vienna, Austria. URL https://www.R-project.org/.

$>$ version

platform x86_64-w64-mingw32

arch x86_64

os mingw 32

system x86_64, mingw32

status

major

minor

year

3

month $\quad 07$

day 05

svn rev $\quad 76782$ 
language $\mathrm{R}$ version.string $R$ version 3.6.1 (2019-07-05)

nickname Action of the Toes

\#Read into R:

$>\mathrm{IP}<-$ read.csv(".../data.csv", sep=",")

\#Confirm dataframe - length/variables and shrink by $24 \mathrm{~m}$ to avoid so-called 'patent cliff':

$>\operatorname{str}(\mathrm{IP})$

'data.frame': 496 obs. of 3 variables:

\$ Date: Factor w/ 496 levels "1/1/1978","1/1/1979",..: 45542841261168209250291332

\$ Number.of.Trademark.Applications: int 266925972552260423862370312627383028 $3088 \ldots$

\$ Number.of.Patent.Applications : int 576058985731663050645439666057996487 $6419 \ldots$

\# Shrinking by 24 months dues to so-called "patent-cliff"

$>$ TrademarksTotal<-IP\$Number.of.Trademark.Applications[1:472]

$>$ PatentsTotal<-IP\$Number.of.Patent.Applications[1:472]

\#Convert to Time-Series, decompose time-series, and perform descriptive statistics

$>$ tsTrademarks<-ts(TrademarksTotal,start $=c(1977,9)$, frequency $=12)$

$>$ tsPatents<-ts(PatentsTotal,start=c(1977,9), frequency=12)

$>$ tsTrademarks

Jan Feb Mar Apr May Jun Jul Aug Sep Oct Nov Dec

1977

2669259725522604

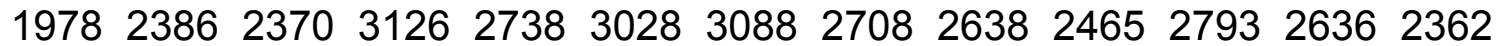

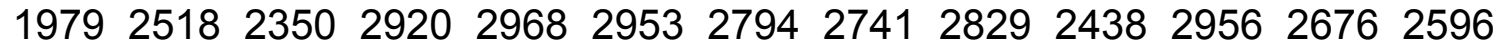

1980246926073035289327973094288325902928408134123559

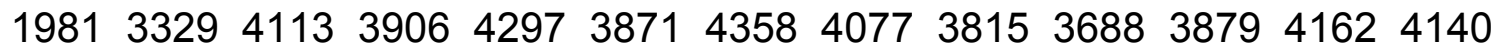

$1982359440095128486845765244 \quad 4942526415843 \quad 189531263529$

1983391535974224429743894543419248934265463441894296

$19844281447251675068476248374914 \quad 48034064489649224474$

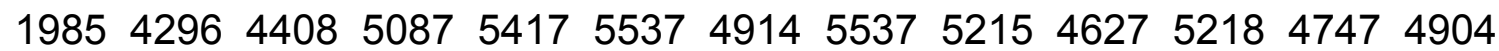

$19864785467755695153539756305152 \quad 51245715478749504931$

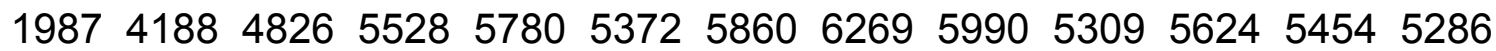

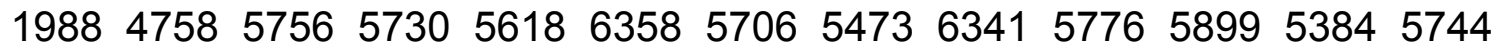

19895546576166156230707164965995656756136687114008450

19909209879710687993698369707931996348167956784177546

1991790679869192973494418962937192608884907388057972

1992748686121024899408776100781001997019348785385999107

19939143914311059113521059411897111601162811448103741124810923

199495991038812173116641229212902107281253611443119321133910634

1995109611185714860123911432313644123251443412631137261292612072

1996125821387215117156361556514433154471559015234162991472713810 
1997140601522916962170791646016465164251536316089169011436715163 1998135101546517624172241580517862175151627016331174261673716897 1999160021843122143209572051524106219992389122563247532422524272 2000234122581530423252042698624831217692462621644229622034017822 2001192481892320732197812077818551183111960814770177271558714181 2002162731635718555189461962217734183591895817639191111648716278 2003161701707719352194611956319045196871861419518207521785718083 2004178482022124380228572085121971207942168620329205452016319727 2005198522115625021231062258323621212832440521769214192183320716 2006215322281027578227502438624861215802531922311240772265820909 2007237742436528236256402746726565255202786023629274072452121614 2008250112568927048275772658624960260862385724425241241907419873 2009191112084923869235732233724084235782262522336237952124821987 2010212212309728105251192395325080227962341323092237392315121794 2011226862423230145268962618727407250242770825636248072455023480 2012242952730529660276602852126754266362779424270273692444722310 2013253822663028633290022960926566276972861226811289912562224625 2014265432724330853307522945028680303932818630059320192621226558 2015271822968135025337863112334043333163132732138325842990930094 2016294613245637317355063486135211320273535633293313943145233063

$>$ tsPatents Jan Feb Mar Apr May Jun Jul Aug Sep Oct Nov Dec

1977

1978506454396660579964876419567158315697601257566210

1979530352406131607162476087572659995541645959136399

1980549256196491597162926325607753286143624654146726

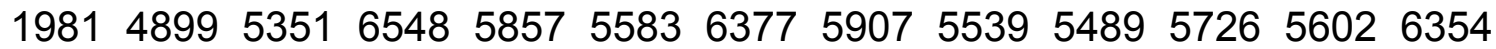

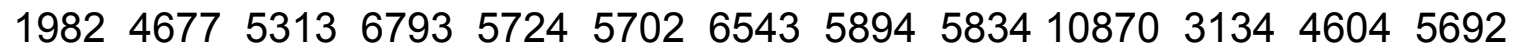

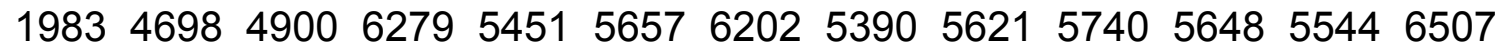

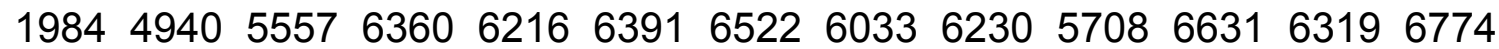

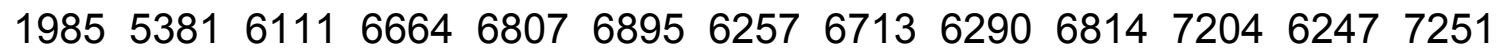

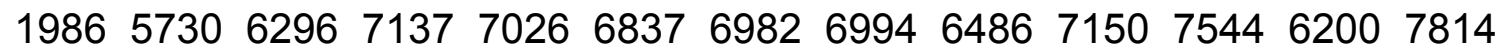

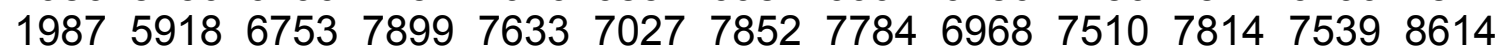

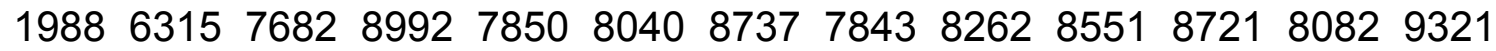

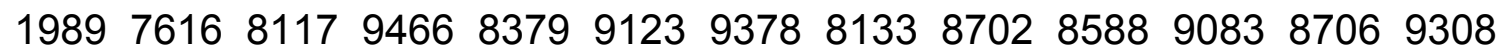

19908151847297079061919793318963926985331044481289421

1991787982709363941394069080939489549327973791559704

19928237848510025959188811028298228666100099586918010693

199380638728110649963924610500979395311023299041003411401

19949243961412007104531089312276103031130012499114441144413760 199510445107161383511945172222812388601034011124109131115212746 1996103461111213067126891300013285136771319514326145241331915858 1997131211353115641152871501516286154561481516658168711452916885 1998127221374616543150621451116769156601442615607156031486118092 1999128651420417997157911533717893159631630817160164471670219417 2000145861645120021159221788419641155211827819273178341853219122 2001164931712721285176921859320122182021975317856192391821019660 2002176941728120278189631989119568192401875419562200341779921302 2003167401689819990189071824219748188301754219825197081689622063 2004155281693121274182121671220593177791794319884175211813022102 
2005149681702122490185461798721090169141846219787175921813322188 2006145501670122216172081940221150175601957119828193121899922766 2007169301753922271184271948020506187972026719143250451819521820 2008167171882521168197621931821035198961854621495214131812023736 2009153751769621848188161743820736191641773620407196031832723801 2010149721769619786201991901922414197601986721604206572061225143 2011172611899225367204282096423822200582226826472206582192626522 2012172221916721736225462392125237230542475627051229292443727708 2013183162372842788195012263422932233502331325042251262301528838 2014193912225330969233872404025581247002308627091251412185529294 2015195992164324767231842254626852237832259025856231592172627049 2016189702093323298199482083722912184172075221299177741779119436

$>$ plot(decompose(tsTrademarks,type="additive"))

$>$ plot(decompose(tsPatents,type="additive"))

\#ldentify outliers

\#Javier López-de-Lacalle (2019). tsoutliers: Detection of Outliers in Time Series. R package version 0.6-8.

\# https://CRAN.R-project.org/package=tsoutliers

library(tsoutliers)

$>$ TrademarksOutliers<-tso(tsTrademarks,types = c("AO","LS","TC"), maxit.iloop=10)

$>$ PatentsOutliers<-tso(tsPatents, types = c("AO","LS","TC"), maxit.iloop=10)

$>$ TrademarksOutliers

Series: tsTrademarks

Regression with $\operatorname{ARIMA}(2,1,1)(0,1,2)[12]$ errors

Coefficients:

ar1 ar2 ma1 sma1 sma2 AO61 LS147 LS262

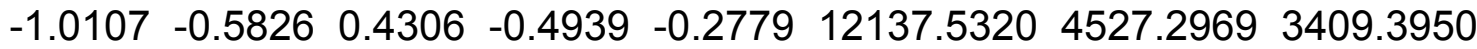

s.e. $0.0834 \quad 0.0440 \quad 0.1067 \quad 0.0480 \quad 0.0458 \quad 669.2913 \quad 681.1637 \quad 674.9746$

sigma^2 estimated as 868751: log likelihood=-3790.79

$\mathrm{AIC}=7599.58 \quad \mathrm{AlCc}=7599.98 \quad \mathrm{BIC}=7636.74$

Outliers:

type ind time coefhat tstat

1 AO 61 1982:09 1213818.135

2 LS 147 1989:11 45276.646

3 LS 262 1999:06 34095.051

$>$ PatentsOutliers

Series: tsPatents

Regression with $\operatorname{ARIMA}(3,0,0)(2,1,2)[12]$ errors

Coefficients:

ar1 ar2 ar3 sar1 sar2 sma1 sma2 AO61 AO214

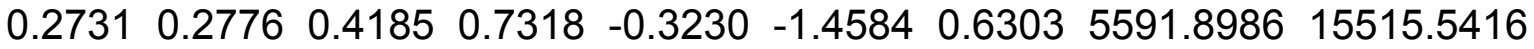

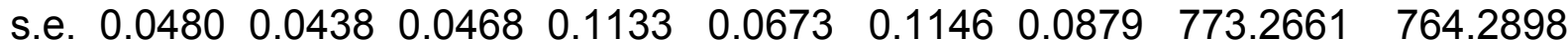
$\mathrm{AO} 362 \mathrm{AO} 427$

5058.304017555 .5353

s.e. 757.5541799 .8064

sigma^2 estimated as 917822: log likelihood=-3812.79

$\mathrm{AlC}=7649.58 \quad \mathrm{AICC}=7650.27 \quad \mathrm{BIC}=7699.15$ 
Outliers:

type ind time coefhat tstat

1 AO $611982: 09 \quad 55927.232$

2 AO 214 1995:06 1551620.301

3 AO 362 2007:10 50586.677

4 AO 427 2013:03 1755621.950

> plot(TrademarksOutliers); X11(); plot(PatentsOutliers)

\#Clean/smooth data - replace identified outliers $(\mathrm{X})$ with average of prior $(\mathrm{X}(\mathrm{t}-1))$ and posterior $(\mathrm{X}(\mathrm{t}+1))$

$>$ Trademarks<-tsTrademarks; Patents<-tsPatents

$>$ Trademarks[61] $=($ Trademarks[62]+Trademarks[64]) $/ 2$

$>$ Trademarks[147] $=($ Trademarks[146]+Trademarks[148] $) / 2$

$>$ Trademarks[262] $=($ Trademarks[261] + Trademarks[263] $) / 2$

$>$ Patents[61] $=($ Patents[62]+Patents[64]) / 2

$>$ Patents[214] $=($ Patents[213]+Patents[215] $) / 2$

$>$ Patents[362] $=($ Patents[361] +Patents[363] $) / 2$

$>$ Patents[427] $=($ Patents[426] + Patents[428] $) / 2$

$>$ plot(decompose (Trademarks,type="additive"))

$>$ plot(decompose(Patents,type="additive"))

> library(moments); citation("moments")

\#Lukasz Komsta and Frederick Novomestky (2015). moments: Moments, cumulants, skewness, kurtosis \#and related tests. R package version 0.14. https://CRAN.R-project.org/ package $=$ moments

\#Use fitted output from tsoutliers

>summary(Trademarks); sd(Trademarks); skewness(Trademarks); kurtosis(Trademarks)

Min. 1st Qu. Median Mean 3rd Qu. Max.

$\begin{array}{llllll}1895 & 5537 & 15456 & 15276 & 23574 & 37317\end{array}$

[1] 9418.054

[1] 0.2020644

[1] 1.763723

>summary(Patents); sd(Patents); skewness(Patents); kurtosis(Patents)

Min. 1st Qu. Median Mean 3rd Qu. Max.

$\begin{array}{llllll}3134 & 7598 & 14468 & 13930 & 19422 & 30969\end{array}$

[1] 6523.347

[1] 0.1854975

[1] 1.757654

\#note: skew/kurtosis comparative - no need to transform

\#auto/serial correlation

acf(Trademarks);pacf(Trademarks) 


\section{Series Trademarks}

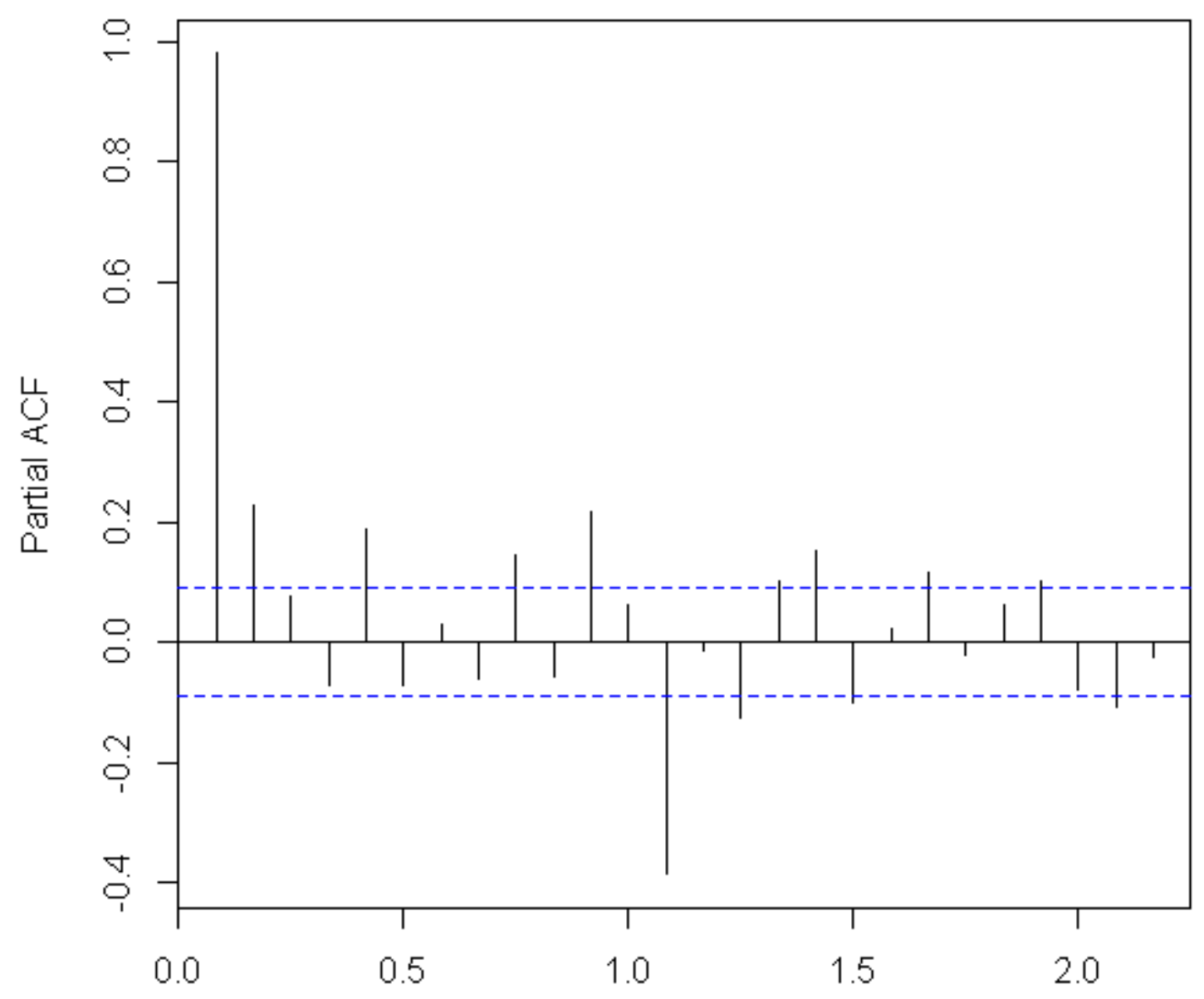

acf(Patents);pacf(Patents)

\#Perform normality, stationarity, seasonality, long-memory, and non-linearity tests $>$ \#normality test

> library(nortest);citation("nortest")

To cite package 'nortest' in publications use: Juergen Gross and Uwe Ligges (2015). nortest: Tests for Normality. R package version 1.0-4. https://CRAN.R-project.org/package=nortest

$>$ ad.test(Trademarks) \#null normality

Anderson-Darling normality test data: Trademarks

$A=11.055, p$-value $<2.2 e-16$

> ad.test(Patents) \#null normality 
Anderson-Darling normality test

data: Patents

$A=13.102, p$-value $<2.2 e-16$

$>$ cvm.test(Trademarks)

Cramer-von Mises normality test

data: Trademarks

$\mathrm{W}=1.7112, \mathrm{p}$-value $=7.37 \mathrm{e}-10$

Warning message:

In cvm.test(Trademarks) : p-value is smaller than 7.37e-10, cannot be computed more accurately

$>$ cvm.test(Patents)

Cramer-von Mises normality test

data: Patents

$\mathrm{W}=2.2197, \mathrm{p}$-value $=7.37 \mathrm{e}-10$

Warning message: In cvm.test(Patents) : p-value is smaller than $7.37 e-10$, cannot be computed more accurately

$>$ \#stationary test

$>$ library(forecast)

>ndiffs(Trademarks, test= "kpss"); ndiffs(Trademarks, test= "adf"); ndiffs(Trademarks, test= "pp")

[1] 1

[1] 1

[1] 1

>ndiffs(Patents, test= "kpss"); ndiffs(Patents, test= "adf"); ndiffs(Patents, test= "pp")

[1] 1

[1] 1

[1] 1

$>$ library(aTSA)

Attaching package: 'aTSA'

The following object is masked from 'package:forecast':forecast

The following object is masked from 'package:graphics': identify

$>$ citation("aTSA")

To cite package 'aTSA' in publications use: Debin Qiu (2015). aTSA: Alternative Time Series Analysis. R package version 3.1.2. https://CRAN.R-project.org/package=aTSA

> stationary.test(Trademarks, method="kpss")

KPSS Unit Root Test alternative: nonstationary

Type 1: no drift no trend

lag stat p.value 
$50.59 \quad 0.1$

Type 2: with drift no trend

lag stat p.value

$50.751 \quad 0.01$

Type 1: with drift and trend

lag stat p.value

50.1810 .023

Note: p.value $=0.01$ means $p$.value $<=0.01$ : .value $=0.10$ means $p$.value $>=0.10$

> stationary.test(Patents, method="kpss")

KPSS Unit Root Test alternative: nonstationary

Type 1: no drift no trend

lag stat p.value

$55.88 \quad 0.01$

Type 2: with drift no trend

lag stat p.value

$55.53 \quad 0.01$

Type 1: with drift and trend

lag stat p.value

$\begin{array}{ll}50.524 & 0.01\end{array}$

Note: p.value $=0.01$ means $p$.value $<=0.01$ : .value $=0.10$ means $p$.value $>=0.10$

\#Now the first diffs

stationary.test(diff(Trademarks), method="kpss")

KPSS Unit Root Test alternative: nonstationary

Type 1: no drift no trend

lag stat p.value

$50.642 \quad 0.1$

Type 2: with drift no trend

lag stat p.value

$50.0294 \quad 0.1$

Type 1: with drift and trend

lag stat p.value

$50.015 \quad 0.1$

Note: p.value $=0.01$ means $p$.value $<=0.01:$. value $=0.10$ means $p$. value $>=0.10$ 
stationary.test(diff(Patents), method="kpss")

KPSS Unit Root Test

alternative: nonstationary

Type 1: no drift no trend

lag stat $p . v a l u e$

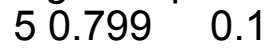

Type 2: with drift no trend

lag stat p.value

$50.0858 \quad 0.1$

Type 1: with drift and trend

lag stat p.value

$50.0716 \quad 0.1$

Note: $p$. value $=0.01$ means $p$.value $<=0.01: p$. value $=0.10$ means $p$. value $>=0.10$

\#Long memory

> library(LongMemoryTS); citation("LongMemoryTS")

To cite package 'LongMemoryTS' in publications use: Christian Leschinski (2019).

LongMemoryTS: Long Memory Time Series. $\mathrm{R}$ package version 0.1.0.

https://CRAN.R-project.org/package=LongMemoryTS

\#Qu Test

$>$ Qu.test(diff(Trademarks),m)

\$W.stat [1] 1.432811

\$CriticalValues

eps $=.02$ eps $=.05$

alpha=.1 $1.118 \quad 1.022$

alpha=.05 $1.252 \quad 1.155$

alpha $=.025 \quad 1.374 \quad 1.277$

alpha=.01 $1.517 \quad 1.426$

$>$ Qu.test(diff(Patents),m)

\$W.stat [1] 2.334971

\$CriticalValues

eps $=.02$ eps $=.05$

alpha=.1 $1.118 \quad 1.022$

alpha=.05 $1.252 \quad 1.155$

alpha=.025 $\quad 1.374 \quad 1.277$

alpha=.01 $1.517 \quad 1.426$

$>$ \#multivariate local Whittle Score

$>$ MLWS(diff(Trademarks), $\mathrm{m}=\mathrm{m}$ )

\$B 
$[1]$,

\$d [1] -0.3782234

\$W.stat [1] 1.502709

\$CriticalValues

alpha $=.1$ alpha $=.05$ alpha $=.025$ alpha $=.01$

$\begin{array}{llll}1.118 & 1.252 & 1.374 & 1.517\end{array}$

$>$ MLWS(diff(Patents), $\mathrm{m}=\mathrm{m}$ )

\$B

$[1]$,

\$d [1] -0.3440901

\$W.stat [1] 2.43958

\$CriticalValues

alpha $=.1$ alpha $=.05$ alpha $=.025$ alpha $=.01$

$\begin{array}{llll}1.118 & 1.252 & 1.374 & 1.517\end{array}$

$>$ \#seasonality tests

> library(seastests); citation("seastests")

To cite package 'seastests' in publications use: Daniel Ollech (2019). seastests: Seasonality Tests. R package version 0.14.2. https://CRAN.R-project.org/package=seastests

> summary(wo(Trademarks))

Test used: WO

Test statistic: 1

P-value: 000

The WO - test identifies seasonality

$>$ summary(wo(Patents))

Test used: WO

Test statistic: 1

P-value: 000

The WO - test identifies seasonality

> $\quad$ isSeasonal(Trademarks,"qs"); $\quad$ isSeasonal(Trademarks,"fried"); isSeasonal(Trademarks,"welch"); 
[1] TRUE

[1] TRUE

[1] TRUE

> isSeasonal(Patents,"qs"); isSeasonal(Patents,"fried"); isSeasonal(Patents, "welch");

[1] TRUE

[1] TRUE

[1] TRUE

\#Nonlinearity Tests

> library(nonlinearTseries); citation("nonlinearTseries")

Attaching package: 'nonlinearTseries' The following object is masked from 'package:aTSA': estimate

The following object is masked from 'package:grDevices': contourLines

To cite package 'nonlinearTseries' in publications use: Constantino A. Garcia (2020). nonlinearTseries: Nonlinear Time Series Analysis. $R$ package version 0.2.10. https://CRAN.R-project.org/package=nonlinearTseries

$>$ nonlinearityTest(Trademarks)

** Teraesvirta's neural network test **

Null hypothesis: Linearity in "mean"

X-squared $=13.65589 \mathrm{df}=2 \mathrm{p}$-value $=0.001083081$

** White neural network test **

Null hypothesis: Linearity in "mean"

X-squared $=14.43927 \mathrm{df}=2 \mathrm{p}$-value $=0.00073207$

** Keenan's one-degree test for nonlinearity **

Null hypothesis: The time series follows some AR process

F-stat $=0.04386686 p$-value $=0.8342031$

** McLeod-Li test **

Null hypothesis: The time series follows some ARIMA process

Maximum $p$-value $=0$

** Tsay's Test for nonlinearity **

Null hypothesis: The time series follows some AR process

F-stat $=3.041166 p$-value $=8.100652 \mathrm{e}-10$

** Likelihood ratio test for threshold nonlinearity **

Null hypothesis: The time series follows some AR process

Alternativce hypothesis: The time series follows some TAR process

X-squared $=51.55873 \mathrm{p}$-value $=0.03278644$

$>$ nonlinearityTest(Patents)

** Teraesvirta's neural network test ** 
Null hypothesis: Linearity in "mean"

X-squared $=110.8611 \mathrm{df}=2 \mathrm{p}$-value $=0$

** White neural network test **

Null hypothesis: Linearity in "mean"

X-squared $=108.337 \mathrm{df}=2 \mathrm{p}$-value $=0$

** Keenan's one-degree test for nonlinearity **

Null hypothesis: The time series follows some AR process

F-stat $=3.379455$-value $=0.06672325$

** McLeod-Li test **

Null hypothesis: The time series follows some ARIMA process

Maximum $p$-value $=0$

** Tsay's Test for nonlinearity **

Null hypothesis: The time series follows some AR process

F-stat $=6.296795 p$-value $=1.162674 \mathrm{e}-15$

** Likelihood ratio test for threshold nonlinearity **

Null hypothesis: The time series follows some AR process

Alternative hypothesis: The time series follows some TAR process

X-squared $=78.45917$ p-value $=2.60603 \mathrm{e}-05$

> library(tsfeatures); citation("tsfeatures")

To cite package 'tsfeatures' in publications use: Rob Hyndman, Yanfei Kang, Pablo Montero-Manso, Thiyanga Talagala, Earo Wang, Yangzhuoran Yang and Mitchell O'Hara-Wild (2020). tsfeatures: Time Series Feature Extraction. R package version 1.0.2. https://CRAN.R-project.org/package=tsfeatures

hurstTrademarks $=0$; hurstPatents $=0$

endT<-length(Trademarks); endP<-length(Patents)

for ( $\mathrm{i}$ in 1 :endT) $\{$ hurstTrademarks[i] <- hurst (Trademarks[1:(1+i*1)]) $\}$

for (i in 1:endP) $\{$ hurstPatents[i] <- hurst (Patents[1:(1+i*1)]) \}

hurstTrademarks $<-$ ts(hurstTrademarks, start $=c(1977,9)$, end $=c(2016,12)$, frequency $=12$ )

hurstPatents<-ts(hurstPatents,start=c(1977,9),end=c(2016,12), frequency=12)

plot(hurstTrademarks); plot(hurstPatents)

> library(tseries);citation("tseries")

'tseries' version: $0.10-47$ 'tseries' is a package for time series analysis and computational finance. See 'library(help="tseries")' for details.

Attaching package: 'tseries' The following objects are masked from 'package:aTSA': adf.test, kpss.test, pp.test

To cite in publications use: 
Adrian Trapletti and Kurt Hornik (2019). tseries: Time Series Analysis and Computational Finance. R package version 0.10-47.

plot(ts.intersect(Patents, hurstPatents), main="'", yax.flip=TRUE)

plot(ts.intersect(Trademarks, hurstTrademarks),main="', yax.flip=TRUE)

plot(ts.intersect(hurstPatents, hurstTrademarks), main="'", yax.flip=TRUE)

\#\#\# End \#\#\# 